\title{
LOCALIZATION FOR RANDOM PERTURBATIONS OF ANISOTROPIC PERIODIC MEDIA
}

\author{
BY \\ Peter StollmanN \\ Fachbereich Mathematik, Johann Wolfgang Goethe-Universität \\ D-60054 Frankfurt am Main, Germany \\ e-mail: stollman@math.uni-frankfurt.de
}

\begin{abstract}
We prove localization for random perturbations of periodic divergence form operators of the form $-\nabla \cdot \mathbf{a}_{\omega} \cdot \nabla$ near the band edges. Here $\mathbf{a}_{\omega}$ is a matrix function which results from an Anderson type perturbation of a periodic matrix function.
\end{abstract}

\section{Introduction, the model, and the results}

In this article we prove Anderson localization for operators of the form $H(\omega)=$ $-\nabla \cdot \mathbf{a}_{\omega} \cdot \nabla$ where $\mathbf{a}_{\omega}$ is a random perturbation of a periodic matrix function. Note that the diffusion equation for a non-homogeneous, anisotropic medium governed by $\mathbf{a}_{\omega}$ is just

$$
\partial_{t} u=H(\omega) u,
$$

so that the spectral properties of $H(\omega)$ have important physical consequences. The application to localization of acoustic waves is explained in the introduction of [4], where the isotropic case (i.e. $\mathbf{a}_{\omega}(x)=\rho^{-1}(x) \cdot I, I$ the unit matrix) is treated. For periodic $\mathbf{a}_{\text {per }}$ one expects $H\left(\mathbf{a}_{\text {per }}\right)$ to have purely absolutely continuous spectrum consisting of closed intervals, the so-called bands, which are separated by open intervals called gaps; see Section 2. For the Anderson-type random perturbations we consider here the spectrum will again have band structure. The spectral type, however, is completely different near the band edges:

Received October 8, 1996 and in revised form June 17, 1997 
there pure point spectrum occurs and the associated eigenfunctions decay exponentially. In a sense this spectral picture is opposite to the situation without the random perturbation, and this despite the fact that the perturbation may very well be small compared to the periodic unperturbed $H\left(\mathbf{a}_{\mathrm{per}}\right)$. Our main result, Theorem 1.1 below, asserts such a spectral picture for models which can be thought of as anisotropic versions of the models investigated by Figotin and Klein in [4]. We follow their line of approach as far as the general scheme of the proof is concerned. Namely, using an adaption of multi-scale analysis, which was designed for the Schrödinger operator case, it remains to prove estimates for restrictions of $H(\omega)$ to finite boxes. The latter have compact resolvent and can thus be analyzed by means of their eigenvalues and eigenfunctions.

We are now going to introduce the model we are working with:

A. Let $\mathrm{a}_{\text {per }}: \mathbb{R}^{d} \rightarrow \mathbb{C}^{d \times d}$ be measurable, bounded, uniformly elliptic (i.e. $\left(\mathbf{a}_{\text {per }}(x) \xi \mid \xi\right) \geq \eta|\xi|^{2}$ a.e. for a fixed $\left.\eta>0\right)$ and $\mathbb{Z}^{d}$-periodic. Then

$$
D\left(h\left(\mathbf{a}_{\mathrm{per}}\right)\right)=W^{1,2}\left(\mathbb{R}^{d}\right), \quad h\left(\mathbf{a}_{\mathrm{per}}\right)[u, v]=\int_{\mathbb{R}^{d}}\left(\mathbf{a}_{\mathrm{per}}(x) \nabla u(x) \mid \nabla v(x)\right) d x
$$

defines a closed, symmetric form whose associated self adjoint operator (see [8]) we denote by $H\left(\mathbf{a}_{\mathrm{per}}\right)$.

Around each site $i \in \mathbb{Z}^{d}$ we choose independently matrices and add them to $\mathbf{a}_{\text {per }}$. To make this precise, we work with a suitable parametrization of the nonnegative matrices: Let

$$
S=[0, \bar{\lambda}]^{d} \times U(d)
$$

where $U(d)$ denotes the unitary matrices and we identify $s=\left(\lambda_{1}, \ldots, \lambda_{d}, u\right) \in S$ with the matrix

$$
u^{*} \operatorname{diag}\left(\lambda_{1}, \ldots, \lambda_{d}\right) u \text {. }
$$

Thus $S$ is a parametrization of all nonnegative matrices with norm less than $\bar{\lambda}$ (where, of course, some matrices are encoded by different $s \in S$, e.g. $\lambda \cdot I$ ). As a probability measure on $S$ we choose

$$
d \mu=g_{1}\left(\lambda_{1}\right) d \lambda_{1} \times \cdots \times g_{d}\left(\lambda_{d}\right) d \lambda_{d} \times d \nu
$$

where $g_{i} \in L^{\infty}$ with support $[0, \bar{\lambda}],\left\|g_{i}\right\|_{1}=1$ and $\nu$ is an arbitrary probability measure on the unitary group, e.g. the Haar measure. For the measure $\mu$ we assume

$$
\mu\left\{s \in S ; \lambda_{1}, \ldots, \lambda_{d} \geq t\right\} \geq 1-t^{\tau} \text { and } \mu\left\{s \in S ; \lambda_{1}, \ldots, \lambda_{d} \leq \bar{\lambda}-t\right\} \geq 1-t^{\tau}
$$


for some $\tau>d$ and small $t>0$. Finally, let $\chi_{i}$ be the characteristic function of the cube of unit sidelength centered at $i \in \mathbb{Z}^{d}$. We define

$$
\begin{gathered}
\Omega=S^{\mathbb{Z}^{d}}, \quad \text { equipped with } \mathbb{P}=\bigotimes_{i \in \mathbb{Z}^{d}} \mu \\
\mathbf{a}_{\omega}=\mathbf{a}_{\mathrm{per}}+\sum_{i \in \mathbb{Z}^{d}} \chi_{i} \omega(i) \quad \text { for } \omega=(\omega(i)) \in \Omega, \\
H(\omega)=H\left(\mathbf{a}_{\omega}\right)
\end{gathered}
$$

where the latter is defined as in (1.1).

An astonishing consequence of the following analysis is the fact that our results depend in no way upon which measure $\nu$ we choose on the unitary group. For instance, one could take the point measure concentrated on $\bar{\lambda} \cdot I$ or any other particular matrix.

Before stating our main result we infer the following formula for the spectrum of $H(\omega)$ (which is deterministic by standard theory of ergodic operators, e.g. $[2,10])$ from Proposition 2.3:

$$
\sigma(H(\omega))=\bigcup_{\lambda \in[0, \bar{\lambda}]} \sigma\left(H\left(\mathbf{a}_{\mathrm{per}}\right)-\lambda \cdot \Delta\right)=: \Sigma \quad \text { for } \mathbb{P} \text { a.e. } \omega
$$

Since $H\left(\mathbf{a}_{\mathrm{per}}\right)-\lambda \cdot \Delta=H\left(\mathbf{a}_{\mathrm{per}}+\lambda \cdot I\right)$ is periodic, $\Sigma$ has band structure. The band edges of $\Sigma$ are "induced" by band edges of $H\left(\mathbf{a}_{\mathrm{per}}\right)$ and $H\left(\mathbf{a}_{\mathrm{per}}+\bar{\lambda} \cdot I\right)$ respectively, and correspond to the rare events that all the eigenvalues of $\omega(i)$ at all the sites $i$ are extremely small respectively extremely large. At these so-called fluctuation boundaries one expects localization. This is the content of our main Theorem:

Theorem 1.1: Assume that $H(\omega)$ is as above. Then in a neighborhood of $\partial \Sigma \backslash\{0\}$ the $H(\omega)$ have pure point spectrum $\mathbb{P}$-a.s. with exponentially decaying eigenfunctions.

Note that we excluded $\inf \Sigma=0$ in the Theorem. The reason is that 0 is not a fluctuation boundary as this value belongs to the spectrum of all finite box hamiltonians with the constant function as eigenfunction and one does not expect localization there (see the discussion in [10], Chapter IV, 8). Moreover let us point out that in contrast to [4], for instance, we do not require any small coupling constant regime.

While it is clear from what was said above that $\Sigma$ consists of a union of closed intervals it is not a priori clear that there are examples exhibiting gaps in the 
spectrum of $H\left(\mathrm{a}_{\mathrm{per}}\right)$ and hence in $\Sigma$ (for $\bar{\lambda}$ small enough). Fortunately, such kinds of examples were provided by Hempel, [5].

As we already remarked above, this theorem extends in a sense recent results by Figotin and Klein, [4], in whose work the random matrices are scalar multiples of the unit matrix. In some instances we have given easier proofs, notably concerning the location of the spectrum of $H\left(\mathbf{a}_{\text {per }}\right)$ and $H\left(\mathbf{a}_{\omega}\right)$. This is mainly due to a simple observation stated in Lemma 2.2 below.

Other related work is the recent joint work [9] of the author together with W. Kirsch and G. Stolz, where localization near the band edges is proven for random perturbations of periodic Schrödinger operators. In particular our proof of the Wegner estimate is, as in [9], modelled along the arguments of [8].

A very nice framework for localization near band edges can be found in the article [1] by Barbaroux, Combes and Hislop. We refer the reader to this paper for a comprehensive list of results concerning localization for additive perturbations. Finally, I thank P.D. Hislop for fruitful discussions and the referee for helpful comments.

\section{The spectrum}

Here we collect some basic facts concerning the spectrum of $H\left(\mathbf{a}_{\mathrm{per}}\right)$ and use them to calculate the deterministic spectrum of $H(\omega)$. Let us start with Floquet theory: consider the unitary

$$
\begin{gathered}
U: L^{2}\left(\mathbb{R}^{d}\right) \rightarrow \int_{[0,2 \pi)^{d}}^{\oplus} L^{2}[0,2 \pi)^{d} \frac{1}{(2 \pi)^{d}} d \theta, \\
U f(x, \theta)=\sum_{k \in \mathbb{Z}^{d}} e^{-k(x+\theta)} f(x+\theta) .
\end{gathered}
$$

It is easy to see that

$$
U H\left(\mathbf{a}_{\mathrm{per}}\right) U^{*}=\int_{[0,2 \pi)^{d}}^{\oplus} T(\theta) \frac{1}{(2 \pi)^{d}} d \theta
$$

where $T(\theta)$ is defined by the associated form

$$
(T(\theta) u \mid v)=\int_{[0,2 \pi)^{d}}\left(\mathbf{a}_{\text {per }}(x)(\nabla-i \theta) u \mid(\nabla-i \theta) v\right) \frac{1}{(2 \pi)^{d}} d x
$$

with form domain

$$
W_{p e r}^{1,2}\left([0,2 \pi)^{d}\right)=\overline{C_{p e r}^{\infty}\left([0,2 \pi)^{d}\right)} W^{1,2}
$$

which can be identified with the Sobolev space over the flat torus. Note that basing the calculations on the forms we don't need to assume any smoothness 
of the matrix $\mathbf{a}_{\text {per }}$. Obviously the $T(\theta)$ have discrete spectrum; denoting their eigenvalues by $E_{n}(\theta), n \in \mathbb{N}$ we have the following representation for the spectrum which is well-known in the Schrödinger operator case (see [11], Section XIII):

$$
\sigma\left(H\left(\mathbf{a}_{\mathrm{per}}\right)\right)=\bigcup_{\theta \in[0,2 \pi)^{d}, n \in \mathbb{N}} E_{n}(\theta) .
$$

Since $T(\theta)$ depends analytically and in particular continuously upon $\theta$ for each fixed $n$ the corresponding $n$-th eigenvalues for different $\theta$ constitute an interval called the $n$-th band. Again by holomorphic dependence on $\theta$ the spectrum of $H\left(\mathbf{a}_{\mathrm{per}}\right)$ will be purely absolutely continuous apart from some discrete eigenvalues, whose existence we cannot exclude. All this is quite standard in the Schrödinger operator case, giving pure absolutely continuous spectrum under mild assumptions concerning the potential; since it is not central to what follows here we refer the reader to the monograph [12] and to the article [6] where related material can be found.

Writing $\Lambda=\Lambda_{l}(i)$ for an open cube with sidelength $l$ centered at $i \in \mathbb{Z}^{d}$ we denote by $H_{\Lambda}(\mathbf{a})$ the operator in $L^{2}(\Lambda)$ associated with the form $h(\mathbf{a})$ defined on $W_{\text {per }}^{1,2}$ by the analog of (1.1). It is clear that periodic boundary conditions are best suited to our model. We have:

Proposition 2.1: For any measurable, bounded, uniformly elliptic matrixvalued function a we have

$$
\sigma(H(\mathbf{a})) \subset \overline{\bigcup_{n \in \mathbb{N}} \sigma\left(H_{\Lambda_{n}(0)}(\mathbf{a})\right)}
$$

If $\mathbf{a}$ is $\mathbb{Z}^{d}$-periodic, equality holds in (2.2).

One possibility to deduce (2.2) is by using Floquet theory and convergence results for operators. We prefer an elementary argument which will be of use later on. It is based upon

LEMMA 2.2: Let $S$ be a nonnegative operator on some Hilbert space; denote by $s$ the form associated with $S$ and let $D \subset D(s)$ be dense with respect to the form norm $\|\cdot\|_{s}$. Then the following are equivalent for $E \in \mathbb{R}$ :

(i) $E \in \sigma(S)$

(ii) There exist normalized $f_{n} \in D$ such that

$$
\sup _{g \in D(s),\|g\|_{s}=1}(s-E)\left[f_{n}, g\right] \rightarrow 0 \quad \text { as } n \rightarrow \infty .
$$


Proof: (i) $\Longrightarrow$ (ii): We find a normalized sequence $\left(g_{n}\right)$ in $D(S)$ with $(S-E) g_{n} \rightarrow$ 0 (either $E$ is an eigenvalue in which case we take the $g_{n}$ to be a fixed eigenfunction or $E$ is in the essential spectrum and there exists a singular sequence, see [15], Thm. 7.24, p. 203). Taking normalized $f_{n}$ in $D$ which approximate $g_{n}$ with respect to the form norm we arrive at (ii).

(ii) $\Longrightarrow$ (i): Assume $E \notin \sigma(S)$. Then $\sup _{n \in \mathbb{N}}\left\|(S-E)^{-1} f_{n}\right\|_{s}=: c<\infty$. This gives

$$
\left\|f_{n}\right\|^{2}=(s-E)\left[f_{n},(S-E)^{-1} f_{n}\right] \leq c \cdot \sup _{g \in D(s),\|g\|_{s}=1}(s-E)\left[f_{n}, g\right] \rightarrow 0
$$

a contradiction.

Note that in the implication (ii) $\Longrightarrow$ (i) above we can even consider sequences of operators and find respectively that $E \in \overline{\bigcup \sigma\left(S_{n}\right)}$. In fact, we will use such an argument in the following proof.

Proof of Proposition 2.1: "C": Let $E \in \sigma(H(\mathbf{a}))$. By Lemma 2.2 we find a sequence $\left(f_{n}\right)$ in $C_{c}^{\infty}$ such that

$$
\sup _{g \in W^{1,2},\|g\|_{W^{1,2}}=1}(h(\mathbf{a})-E)\left[f_{n}, g\right] \rightarrow 0 \quad \text { as } n \rightarrow \infty
$$

using that the form norm of $h(\mathbf{a})$ is equivalent to the Sobolev norm. For $l_{n} \in \mathbb{N}$ large enough the support of $f_{n}$ is contained in $\Lambda_{l_{n}}(0)$ and consequently $f_{n}$ lies in the form domain of $H_{\Lambda_{l_{n}}(0)}$. Assume now that $E \notin \overline{\bigcup_{n \in \mathbb{N}} \sigma\left(H_{\Lambda_{n}(0)}(\mathbf{a})\right)}$. Then there is a minimal positive distance of $E$ to $\overline{\bigcup_{n \in \mathbb{N}} \sigma\left(H_{\Lambda_{l_{n}}(0)}(\mathbf{a})\right)}$ which implies that the corresponding resolvents are uniformly bounded. This gives

$$
\begin{aligned}
\left\|f_{n}\right\|^{2} & =(h(\mathbf{a})-E)\left[f_{n},\left(H_{\Lambda_{l_{n}}(0)}-E\right)^{-1} f_{n}\right] \\
& \leq c \cdot \sup _{g \in W^{1,2},\|g\|_{W^{1,2}=1}}(h(\mathbf{a})-E)\left[f_{n}, g\right] \rightarrow 0,
\end{aligned}
$$

a contradiction. For the last inequality we use that each $\left(H_{\Lambda_{l_{n}}(0)}-E\right)^{-1} f_{n}$ which is a priori defined only on the cube can be extended to a $W^{1,2}$-function on all of $\mathbb{R}^{d}$ in such a way that the norms stay bounded. The norms of $\left(H_{\Lambda_{l_{n}}(0)}-E\right)^{-1} f_{n}$ are bounded in $n$ because of the uniform boundedness of the resolvents mentioned above.

Let us now prove the converse inclusion in case that $\mathbf{a}$ is periodic. Here we use a well-known strategy of constructing singular sequences. It suffices to prove that every $E \in \sigma\left(H_{\Lambda_{k}(0)}(\mathbf{a})\right)$ is contained in $\sigma(H(\mathbf{a}))$, since the latter is a closed set. Take $f$, a normalized eigenfunction of $H_{\Lambda_{k}(0)}(\mathrm{a})$ with eigenvalue $E$ and extend 
it periodically to $\Lambda_{n \cdot k}(0)$. Note that this shows that

$$
\sigma\left(H_{\Lambda_{k}(0)}(\mathbf{a})\right) \subset \sigma\left(H_{\Lambda_{n \cdot k}(0)}(\mathbf{a})\right) .
$$

Use a $C^{1}$ cut-off function $\phi_{n}$ which takes values in the unit interval, has support in $\Lambda_{n \cdot k}(0)$ and is 1 on the cube $\Lambda_{(n-2) \cdot k}(0)$. Consider $g_{n}:=\phi_{n} \cdot f$ and note that

$$
(n-2)^{d} \leq\left\|g_{n}\right\|^{2} \leq n^{d} \quad \text { and } \quad C(n-2)^{d} \leq\left\|g_{n}\right\|_{W^{1,2}}^{2} \leq C \cdot n^{d},
$$

and that the gradient of $\phi_{n}$ is supported on a set of measure proportional to $n^{d-1}$. Using these properties and plugging $f_{n}:=\left\|g_{n}\right\|^{-1} \cdot g_{n}$ into the definition of $h(\mathbf{a})$ one verifies in a straightforward calculation that $f_{n}$ obey the condition required in Lemma 2.2 (ii).

Proposition 2.3: Assume A. Then

$$
\sigma(H(\omega))=\bigcup_{\lambda \in[0, \bar{\lambda}]} \sigma\left(H\left(\mathbf{a}_{\mathrm{per}}\right)-\lambda \cdot \Delta\right) \quad \text { for } \mathbb{P} \text { a.e. } \omega .
$$

Proof: " $C$ " follows from the min-max principle and Proposition 2.1 since

$$
H_{\Lambda}\left(\mathbf{a}_{\text {per }}\right) \leq H_{\Lambda}\left(\mathbf{a}_{\omega}\right) \leq H_{\Lambda}\left(\mathbf{a}_{\text {per }}+\bar{\lambda} \cdot I\right)
$$

for all cubes $\Lambda$.

" $\supset$ ": Given a $\lambda \in[0, \bar{\lambda}]$ and an $E \in \sigma\left(H\left(\mathbf{a}_{\text {per }}+\lambda \cdot I\right)\right.$ we find a singular sequence $\phi_{n}$ in $C_{c}^{\infty}$ for $E$ by Lemma 2.2. Let $l_{n} \in \mathbb{N}$ be such that $\operatorname{supp}\left(\phi_{n}\right) \subset \Lambda_{l_{n}}(0)$, and for $x \in \mathbb{Z}^{d}$ consider

$$
\Omega_{n}(x):=\left\{\omega \in \Omega ; \omega(k)_{j} \in[\lambda-1 / n, \lambda+1 / n] \text { for all } k \in \Lambda_{l_{n}}(x), j=1, \ldots, d\right\} .
$$

Then $\mathbb{P}\left(\Omega_{n}(x)\right)$ is independent of $x$ and strictly positive. Also, if $\Lambda_{\ell_{n}}(x) \cap \Lambda_{\ell_{n}}(y)=$ $\emptyset$, then $\Omega_{n}(x)$ and $\Omega_{n}(y)$ are independent. Thus, for a.e. $\omega \in \Omega$ there exists an $x_{n}(\omega)$ such that $\omega \in \Omega_{n}\left(x_{n}(\omega)\right)$. This implies

$$
\sup _{g \in W^{1,2},\|g\|_{1,2} \leq 1}\left(h\left(\mathbf{a}_{\omega}\right)-E\right)\left[\phi_{n}\left(\cdot-x_{n}(\omega)\right), g\right] \rightarrow 0 \quad \text { as } n \rightarrow \infty,
$$

i.e. $\phi_{n}\left(\cdot-x_{n}(\omega)\right)$ is a singular sequence for $E$ to $h\left(\mathbf{a}_{\omega}\right)$ in the sense of Lemma 2.2. This concludes the proof of (1.3).

The simple formula (1.3) can be extended to the case where $\chi_{i}$ is replaced by a more general cut-off function. In fact, for $0 \leq \phi \leq 1, \phi_{i}:=\phi(x-i)$ instead of $\chi_{i}$ one has

$$
\sigma(H(\omega))=\bigcup_{\lambda \in[0, \bar{\lambda}]} \sigma\left(H\left(\mathbf{a}_{\text {per }}+\lambda \cdot \sum_{i \in \mathbb{Z}^{d}} \phi_{i} \cdot I\right)\right)
$$


without any significant change in the proof. Note, however, that in the sequel we would need

$$
\sum_{i \in \mathbb{Z}^{d}} \phi_{i} \geq \alpha>0
$$

\section{The Wegner estimate}

The estimate we are aiming at is one of the basic ingredients of multi-scale analysis. It is again phrased in terms of the box hamiltonians $H_{\Lambda}\left(\mathbf{a}_{\omega}\right)$ and states that it is quite unlikely to hit a fixed energy with the spectrum of the box hamiltonian when varying the $\omega$. The precise interplay of probability estimates and size of the box is, however, crucial.

Theorem 3.1 (Wegner's estimate): For every $a>0$ there exists $c_{a}$ such that for all $E, \varepsilon>0$ satisfying $E-\varepsilon>a$ and all $\Lambda=\Lambda_{l}(k)$ with $l \in \mathbb{N}, k \in \mathbb{Z}^{d}$ :

$$
\mathbb{P}\left\{\operatorname{dist}\left(\sigma\left(H_{\Lambda}\left(\mathbf{a}_{\omega}\right)\right), E\right) \leq \varepsilon\right\} \leq c_{a} \cdot \varepsilon \cdot|\Lambda|^{2} .
$$

The proof given here is very similar to the one in [8], which was also employed in [9]. In the latter article we allow for random perturbations with small support which requires considerably more effort. Moreover, in the latter case the Wegner estimate only holds in a neighborhood of the boundary of $\Sigma$, quite in contrast to our theorem above which provides an estimate away from the stable boundary 0 .

Unfortunately, we only obtain a bound quadratic in the volume of the cube and therefore no information about the integrated density of states.

Proof: Choose a monotone $C^{1}$-function $\rho: \mathbb{R} \rightarrow[0,1]$ which is 1 on $[\varepsilon, \infty)$ and 0 on $(-\infty,-\varepsilon]$. We can estimate

$$
\begin{aligned}
\mathbb{P}\left\{\operatorname{dist}\left(\sigma\left(H_{\Lambda}\left(\mathbf{a}_{\omega}\right)\right), E\right) \leq \varepsilon\right\} & \leq \mathbb{E}\left\{\operatorname{tr} \chi_{[E-\varepsilon, E+\varepsilon]}\left(H_{\Lambda}\left(\mathbf{a}_{\omega}\right)\right)\right\} \\
& \leq \mathbb{E}\left\{\operatorname{tr}\left[\rho\left(H_{\Lambda}\left(\mathbf{a}_{\omega}\right)-E+2 \varepsilon\right)-\rho\left(H_{\Lambda}\left(\mathbf{a}_{\omega}\right)-E-2 \varepsilon\right)\right]\right\} \\
& \leq \mathbb{E}\left\{\operatorname{tr}\left[\int_{-2 \varepsilon}^{2 \varepsilon} \rho^{\prime}\left(H_{\Lambda}\left(\mathbf{a}_{\omega}\right)-E+t\right) d t\right]\right\} \\
& =\sum_{n} \int_{-2 \varepsilon}^{2 \varepsilon} d t \prod_{j \in \Lambda} \int_{S} d \mu(\omega(j)) \rho^{\prime}\left(E_{n}\left(H_{\Lambda}\left(\mathbf{a}_{\omega}\right)\right)-E+t\right),
\end{aligned}
$$

where $E_{n}$ denotes the $n$-th eigenvalue (counted with multiplicity) of an appropriate operator. In order to calculate this expression further, let us introduce a more convenient notation; since $H_{\Lambda}\left(\mathbf{a}_{\omega}\right)$ only depends upon the $\omega(k)$ with $k \in \Lambda$ 
we write $(\omega(k))_{k \in \Lambda}=\left(x^{(k)}, u^{(k)}\right)_{k \in \Lambda}$, where $x^{(k)} \in[0, \bar{\lambda}]^{d}$ and $u^{(k)}$ is a unitary matrix. With the obvious notation let us regard

$$
\frac{\partial}{\partial x_{i}^{(k)}} E_{n}(H(x, u))
$$

for $k \in \Lambda, i=1, \ldots, d$. Writing $\varepsilon_{i i}$ for the matrix $\left(\delta_{k i} \delta_{i l}\right)_{k, l=1}^{d}$ and using the Hellmann-Feynman theorem [14], p. 151, we get

$$
\frac{\partial}{\partial x_{i}^{(k)}} E_{n}(H(x, u))=\left(\chi_{k} u^{(k)} \varepsilon_{i i} u^{(k) *} \nabla \psi \mid \nabla \psi\right)
$$

where $\psi$ is a normalized eigenfunction of $H(x, u)$ with eigenvalue $E_{n}(x, u)$. Denote

$$
M:=\sup \left\{\left\|\mathbf{a}_{\omega}(x)\right\| ; x \in \mathbb{R}^{d}, \omega \in \Omega\right\},
$$

where $\left\|\mathbf{a}_{\omega}(x)\right\|$ stands for the operator norm of the matrix, so that

$$
(H(\omega) \phi \mid \phi)=\left(\mathbf{a}_{\omega} \nabla \phi \mid \nabla \phi\right) \leq M \cdot\|\nabla \phi\|^{2} \text {. }
$$

Consequently,

$$
\sum_{k \in \Lambda} \sum_{i=1}^{d} \frac{\partial}{\partial x_{i}^{(k)}} E_{n}(H(x, u))=\|\nabla \psi\|^{2} \geq M^{-1}(H(x, u) \psi \mid \psi) \geq M^{-1} \cdot E_{n}(H(x, u)) .
$$

Since the eigenvalues in the last inequality are all bounded below by $a$ we arrive at

$$
\begin{aligned}
a \cdot M^{-1} \cdot \rho^{\prime}\left(E_{n}(\cdot)\right) & \leq \rho^{\prime}\left(E_{n}(\cdot)\right) \cdot \sum_{k \in \Lambda} \sum_{i=1}^{d} \frac{\partial}{\partial x_{i}^{(k)}} E_{n}(H(x, u)) \\
& =\sum_{k \in \Lambda} \sum_{i=1}^{d} \frac{\partial}{\partial x_{i}^{(k)}} \rho\left(E_{n}(H(x, u))-E+t\right)
\end{aligned}
$$

so we can proceed to estimate the rhs of $(3.1)$ by

$$
\begin{gathered}
\cdots \leq M a^{-1} \sum_{n} \int_{-2 \varepsilon}^{2 \varepsilon} d t \prod_{j \in \Lambda} \int_{S} d \mu(\omega(j))\left(\sum_{k \in \Lambda} \sum_{i=1}^{d} \frac{\partial}{\partial x_{i}^{(k)}} \rho\left(E_{n}(H(x, u))-E+t\right)\right) \\
=M a^{-1} \sum_{n} \int_{-2 \varepsilon}^{2 \varepsilon} d t \prod_{j} \int_{U(d)} d \nu\left(u^{(j)}\right) \prod_{l} \int_{0}^{\bar{\lambda}} d x_{l}^{(j)} g_{l}\left(x_{l}^{(j)}\right) \cdots \\
\ldots\left(\sum_{k \in \Lambda} \sum_{i=1}^{d} \frac{\partial}{\partial x_{i}^{(k)}} \rho\left(E_{n}(H(x, u))-E+t\right)\right)
\end{gathered}
$$




$$
\begin{gathered}
=M a^{-1} \sum_{n} \int_{-2 \varepsilon}^{2 \varepsilon} d t \sum_{k \in \Lambda} \sum_{i=1}^{d} \cdots \\
\left.\cdots\left(\prod_{j} \int_{U(d)} d \nu\left(u^{(j)}\right) \prod_{l} \int_{0}^{\bar{\lambda}} d x_{l}^{(j)} g_{l}\left(x_{l}^{(j)}\right) \frac{\partial}{\partial x_{i}^{(k)}} \rho\left(E_{n}(H(x, u))-E+t\right)\right)\right) .
\end{gathered}
$$

For fixed $k, i$ we can estimate the inner integral by

$$
\left.\left.\left(\cdots \prod_{(j, l) \neq(k, i)} \int_{0}^{\bar{\lambda}} d x_{l}^{(j)} g_{l}\left(x_{l}^{(j)}\right) \int d x_{i}^{(k)} \frac{\partial}{\partial x_{i}^{(k)}} \rho\left(E_{n}(H(x, u))-E+t\right)\right)\right)\left\|g_{i}\right\|_{\infty}\right)
$$

since $\rho$ was supposed to be monotone. The inner integral can be calculated as

$$
\rho\left(E_{n}\left(H\left(x^{\prime}, x_{l}^{(k)}=\bar{\lambda}, u\right)\right)-E+t\right)-\rho\left(E_{n}\left(H\left(x^{\prime}, x_{l}^{(k)}=0, u\right)\right)-E+t\right)
$$

where the ' indicates the $x$-coordinates different from $x_{l}^{(k)}$. Observe that we have $d \cdot|\Lambda|$ terms in the sum over $k, i$. The assertion of the Theorem is proved, if we can check that

$$
\begin{gathered}
\sum_{n} \int_{-2 \varepsilon}^{2 \varepsilon} d t \rho\left(E_{n}\left(H\left(x^{\prime}, x_{l}^{(k)}=\bar{\lambda}, u\right)\right)-E+t\right)-\rho\left(E_{n}\left(H\left(x^{\prime}, x_{l}^{(k)}=0, u\right)\right)-E+t\right) \cdots \\
\cdots \leq C \varepsilon \cdot|\Lambda|
\end{gathered}
$$

but this follows from Weyl's law as $\rho \leq 1$ and only those $n$ with energy below $E+2 \varepsilon$ give a nonzero contribution.

\section{Initial length scale estimates}

In this section we provide the initial length scale estimate which constitute the second main ingredient (along with Wegner's estimate) needed for a multi-scale analysis.

Proposition 4.1: Assume A and let $E \in \partial \Sigma \backslash\{0\}$. Then for $\xi<\tau-d$ there is $\beta>0$ and $l_{0}=l_{0}(d, \xi, \tau, \beta)$ such that

$$
\mathbb{P}\left\{\operatorname{dist}\left(\sigma\left(H_{\Lambda_{l}}\right), E\right) \leq l^{\beta-1}\right\} \leq l^{-\xi}
$$

for all $l \geq l_{0}$.

Proof: For definiteness, let $E \in \partial \Sigma \backslash\{0\}$ be a lower band edge. We fix $h>0$ and consider

$$
\Omega_{l, h}:=\left\{\omega \in \Omega ; \omega_{j, 1}, \ldots, \omega_{j, d} \geq h \quad\left(j \in \Lambda_{l}(0)\right)\right\}
$$


Since $E \in \partial \Sigma$ we find $\delta>0$ such that for $\Lambda=\Lambda_{l}(0)$

$$
\sigma\left(H_{\Lambda}(\omega)\right) \cap[E-\delta, E)=\emptyset .
$$

Obviously, for $\omega \in \Omega_{l, h}$ we have

$$
H_{\Lambda}(\omega) \geq H_{\Lambda}(\mathbf{a})-h \Delta_{\Lambda}
$$

so that

$$
\inf \left(\sigma\left(H_{\Lambda}(\omega)\right) \cap[E-\delta, \infty)\right) \geq \inf \left(\sigma\left(H_{\Lambda}(\mathbf{a})-h \Delta_{\Lambda}\right) \cap[E-\delta, \infty)\right) \geq E .
$$

As $\sigma\left(H_{\Lambda}(\omega)\right) \cap[E-\delta, E)=\emptyset$ this means that the distance from $E$ to $\sigma\left(H_{\Lambda}(\omega)\right)$ is just the distance of $E$ to the part of the spectrum which lies right to $E$ (whenever it is less then $\delta)$, i.e. $\operatorname{dist}\left(\sigma\left(H_{\Lambda}(\omega)\right) \cap[E, \infty), E\right)$ and thus

$$
\operatorname{dist}\left(\sigma\left(H_{\Lambda}(\omega)\right), E\right) \geq \inf \left(\sigma\left(H_{\Lambda}(\mathbf{a})-h \Delta_{\Lambda}\right) \cap[E-\delta, \infty)\right)-E
$$

for $h>0$ small enough. We will now show that the rhs of inequality (4.2) is bounded below by $h \cdot M^{-1} \cdot E$ where $M$ is the upper bound of $\mathbf{a}_{\omega}$ as defined in the proof of Theorem 3.1. In fact, consider

$$
[0, h] \ni t \mapsto H_{\Lambda}(\mathbf{a})-t \Delta_{\Lambda}
$$

which constitutes an increasing analytic family of type (a) in the sense of Kato (see [7], VII, §2). Hence, for any eigenvalue $E_{n}\left(H_{\Lambda}(\mathbf{a})-h \Delta_{\Lambda}\right)$ of $H_{\Lambda}(\mathbf{a})-h \Delta_{\Lambda}$ above $E$ the $n$-th eigenvalue $E(t)=E_{n}\left(H_{\Lambda}(\mathbf{a})-t \Delta_{\Lambda}\right)$ is an increasing, piecewise analytic function with $E(0) \geq E$. For $s \in[0, h]$ we have by the FeynmanHellmann theorem, [14], p. 151, that

$$
\left.\frac{\partial E(t)}{\partial t}\right|_{t=s}=\left(-\Delta_{\Lambda} \Phi(s) \mid \Phi(s)\right)
$$

where $\Phi(s)$ is a suitable normalized eigenfunction of $H_{\Lambda}(\mathbf{a})-s \Delta_{\Lambda}$ with eigenvalue $E(s)$. Consequently, in inequality (4.3) we may estimate

$$
\cdots \geq M^{-1}\left(\left(H_{\Lambda}(\mathbf{a})-s \Delta_{\Lambda}\right) \Phi(s) \mid \Phi(s)\right) \geq M^{-1} E(s) .
$$

Writing $E(t)$ as the integral over its derivative and using the last estimate we get

$$
E(h) \geq E+M^{-1} E \cdot h
$$

and this implies

$$
\operatorname{dist}\left(\sigma\left(H_{\Lambda}(\omega)\right), E\right) \geq M^{-1} E \cdot h
$$


for $\omega \in \Omega_{l, h}$. On the other hand,

$$
\begin{aligned}
\mathbb{P}\left(\Omega_{l, h}\right) & =1-|\Lambda| \mu\left\{\left(\lambda_{1}, \ldots, \lambda_{d}\right) ; \exists j=1, \ldots, d: \quad \lambda_{j} \leq h\right\} \\
& \geq 1-l^{d} \cdot h^{\tau}
\end{aligned}
$$

by our assumption concerning the single site measure $\mu$. Since $\tau-d>\xi$ we can choose $\beta>0$ small enough to have $\tau \cdot(1-\beta)-d>\xi$. For $h=M E^{-1} l^{\beta-1}$ we arrive at

$$
l^{d} \cdot h^{\tau}=\left(M E^{-1}\right)^{\tau} \cdot l^{-(\tau \cdot(1-\beta)-d)},
$$

so that

$$
\mathbb{P}\left(\Omega_{l, h}\right) \geq 1-l^{-\xi}
$$

for large enough $l$.

If $E$ is an upper band edge we use the same basic idea, this time with an event of the form

$$
\Omega_{l, h}:=\left\{\omega \in \Omega ; \omega_{j, 1}, \ldots, \omega_{j, d} \leq \bar{\lambda}-h \quad\left(j \in \Lambda_{l}\right)\right\}
$$

With standard Combes-Thomas methods ([3]) one finds that for suitable $A, B \subset \Lambda$ one has

$$
\left\|\chi_{A}\left(H_{\Lambda}(\omega)-z\right)^{-1} \chi_{B}\right\| \leq C \cdot \exp (-\gamma \cdot \operatorname{dist}(A, B))
$$

with $\gamma$ proportional to $\operatorname{dist}\left(z, \sigma\left(H_{\Lambda}(\omega)\right)\right)$. For $\Lambda=\Lambda_{l}(x)$ denote

$$
\chi_{\Lambda}^{\bullet}:=\chi_{\Lambda_{l / 3}(x)}, \chi_{\Lambda}^{\mathrm{o}}:=\chi_{\Lambda_{l}(x) \backslash \Lambda_{l-2}(x)} .
$$

The notation is meant to express that one localizes near the center and the boundary of the cube, respectively. From what was said above and Proposition 4.1 one has immediately:

Theorem 4.2: Assume A and let $E \in \partial \Sigma \backslash\{0\}$. Then for $\xi<\tau-d$ there is $\beta>0$ and $l_{0}=l_{0}(d, \xi, \tau, \beta)$ such that for $l \geq l_{0}$ and $I:=\left[E-l^{\beta-1}, E+l^{\beta-1}\right]$

$$
\mathbb{P}\left\{\left\|\chi_{\Lambda}^{\circ}\left(H_{\Lambda}(\omega)-z\right)^{-1} \chi_{\Lambda}^{\bullet}\right\| \leq \exp \left(-l^{\beta}\right) \text { for all } z \in I\right\} \geq 1-l^{-\xi} \text {. }
$$




\section{Multi-scale analysis and localization}

In this section we outline the proof of Theorem 1.1 which is analogous to the one given by Figotin and Klein in [4]. Since it is merely a notational adaption of what can be found in the latter article we sketch the line of reasoning rather than supplying all the details.

An induction on increasing length scales, the so-called multi-scale analysis, is the heart of the matter: in this induction argument one needs the Wegner estimate and the starting point is the initial length scale estimate, Theorem 4.2. The multi-scale analysis then provides exponential decay for the resolvents of the box hamiltonians on larger and larger boxes which can be used to prove exponential decay for generalized eigenfunctions of the operator $H\left(\mathbf{a}_{\omega}\right)$ for a.e. $\omega$ and energies near $\partial \Sigma \backslash\{0\}$. This ends the proof since second-order divergenceform operators of the type considered here admit an expansion in generalized eigenfunctions by the arguments in [4], B.2.

The only other point we have to check is, whether a basic tool, called SimonLieb inequality in [4], extends to the more general framework considered in the present paper. More precisely one has to obtain the following estimate:

(SLI). For any bounded $I_{0} \subset \mathbb{R}$ there is $C=C\left(I_{0}\right)$ such that for all cubes $\Lambda \subset \Lambda^{\prime}$ and subsets $A \subset \Lambda_{l / 3}(x), B \subset \Lambda^{\prime} \backslash \Lambda$ we have

$$
\left\|\chi_{B}\left(H_{\Lambda}(\omega)-z\right)^{-1} \chi_{A}\right\| \leq C \cdot\left\|\chi_{B}\left(H_{\Lambda^{\prime}}(\omega)-z\right)^{-1} \chi_{\Lambda}^{\circ}\right\| \cdot\left\|\chi_{\Lambda}^{\circ}\left(H_{\Lambda}(\omega)-z\right)^{-1} \chi_{A}\right\|
$$

for all $z \in I_{0}$ and $\omega \in \Omega$.

By standard estimates for weak solutions (see [4], Section B.1) of second-order pde this follows rather straightforwardly.

We end by collecting some remarks:

- In contrast to [4] we stated our main result without an additional coupling constant in front of the random perturbation. To see that we can nevertheless use the multi-scale technique from [4] we just have to notice that the small coupling constant regime is not needed in that respect. In [4] it is rather used to force gaps of $H\left(\mathbf{a}_{\text {per }}\right)$ to remain open. Our formulation using the formula for $\Sigma$ follows a somewhat different philosophy which seems to be advantageous. Once you have gaps in $\Sigma$ you can apply Theorem 1.1; if not, you can modify the underlying model using a coupling constant to produce gaps (whenever you started with $H\left(\mathbf{a}_{\text {per }}\right)$ which exhibits gaps). 
- There is a special situation not covered by Theorem 1.1 in which our method gives localization. Namely, if $H\left(\mathbf{a}_{\text {per }}\right)$ has a gap which is just filled by the random perturbation, i.e. if there is

$$
E \in \Sigma \backslash \bigcup_{\lambda \in(0, \bar{\lambda})} \sigma\left(H\left(\mathbf{a}_{\mathrm{per}}+\lambda \cdot I\right)\right) .
$$

In this case it is not hard to prove the initial length scale estimate near $E$.

- In our framework as described in $\mathbf{A}$ the different eigenvalues of the randomly chosen perturbing matrices are required to be independent. Thus, on a formal level, the isotropic case considered in [4] is not covered by the above results. To do so, one has to look at the simpler model $S=[0, \bar{\lambda}] \cdot I$ to which our methods apply.

- During the induction process in which one proves the existence of a small interval of localization one unfortunately looses control over the length of this interval so that quantitative results in this direction seem to be out of reach at the moment.

- Very recently I obtained an easy geometric proof [13] of a substantial generalization of Theorem 3.1 above. It gives, in particular, localization for the model introduced in $\mathbf{A}$ with much more general distribution of the eigenvalues. For instance, it can be applied to the case where $\mu$ is merely required to be of the form

$$
\mu=\gamma_{1} \times \cdots \times \gamma_{d} \times \nu
$$

where the $\gamma_{i}, i=1, \ldots, d$ are probability measures with support $[0, \bar{\lambda}]$ which satisfy a Hölder condition, i.e. are such that $\gamma_{i}(I) \leq|I|^{\alpha}$ for some positive $\alpha$ and every interval $I$. Details will appear elsewhere.

\section{Reference}

[1] J. M. Barbaroux, J. M. Combes and P. D. Hislop, Localization near band edges for random Schrödinger operators, Helvetica Physica Acta 70 (1997), 16-43.

[2] R. Carmona and J. Lacroix, Spectral Theory of Random Schrödinger Operators, Birkhäuser, Boston, Basel, Berlin, 1990. 
[3] J. M. Combes and L. Thomas, Asymptotic behavior of eigenfunctions of multiparticle Schrödinger operators, Communications in Mathematical Physics 34 (1973), 439-482.

[4] A. Figotin and A. Klein, Localization of classical waves I: Acoustic waves, Communications in Mathematical Physics 180 (1996), 439-482.

[5] R. Hempel, Second order perturbations of divergence type operators with a spectral gap, in Symposium on Operator Calculus and Spectral Theory, Lambrecht 1991 (M. Demuth, B. Gramsch and B.-W- Schulze, eds.), Operator Theory Advances and Applications Vol. 57, Birkhäuser, Basel, Boston, Berlin, 1992.

[6] R. Hempel and I. Herbst, Bands and gaps for periodic magnetic Hamiltonians, in Partial Differential Operators and Mathematical Physics, International Conference in Holzhau, 1994 (M. Demuth and B.-W. Schulze, eds.), Operator Theory Advances and Applications Vol. 78, Birkhäuser, Basel, Boston, Berlin, 1995.

[7] T. Kato, Perturbation Theory for Linear Operators, 2nd ed., Springer-Verlag, Berlin, 1976.

[8] W. Kirsch, Wegner estimates and Anderson localization for alloy-type potentials, Mathematische Zeitschrift 221 (1996), 507-512.

[9] W. Kirsch, P. Stollmann and G. Stolz, Localization for random perturbations of periodic Schrödinger Operators, preprint.

[10] L. Pastur and A. Figotin, Random Schrödinger operators, Springer-Verlag, Berlin, 1992.

[11] M. Reed and B. Simon, Methods of Modern Mathematical Physics IV, Academic Press, New York, 1978.

[12] G. V. Rozenblum, M. A. Shubin and M. Z. Solomyak, Spectral theory of differential operators, in Partial Differential Equations (M. A. Shubin, ed.), Encyclopedia of Mathematical Sciences Vol. 64, Springer-Verlag, Berlin, Heidelberg, 1994.

[13] P. Stollmann, A short proof of a Wegner estimate and localization, preprint.

[14] W. Thirring, A Course in Mathematical Physics 3, Quantum Mechanics of Atoms and Molecules, Springer-Verlag, New York, Wien, 1981.

[15] J. Weidmann, Linear Operators in Hilbert Spaces, Graduate Texts in Mathematics Vol. 68, Springer-Verlag, Berlin, 1980.

Ps-files of preprints [9] and [13] can be downloaded from the authors homepage at www.math.uni-frankfurt.de/ stollman. 